\title{
Jens Gudel \\ Schule im Jugendstrafvollzug: Überlegungen und Untersuchungen zu ihrer Ausrichtung als Instrument im Rahmen der Prävention
}

\section{A. Einleitung}

„Du hast zwar keine Chance, aber nutze sie. “1 Dieses paradoxe Zitat des bayerischen Schriftstellers und Filmproduzenten Herbert Achternbusch trifft auch auf die Situation im bundesdeutschen Jugendvollzug zu: Auf der einen Seite sprechen viele Vollzugsfachleute vom Jugendvollzug als einem Chancenvollzug, in dem die Jugendstrafgefangenen zur Mitwirkung am Erreichen ihrer Vollzugsziele und somit zum Ergreifen der angebotenen Chancen motiviert werden sollen. ${ }^{2}$ Angestoßen durch das Urteil des Bundesverfassungsgerichts zur gesetzlichen Verankerung des Jugendstrafvollzuges ${ }^{3}$ legen die einzelnen Bundesländer in ihren Justizvollzugsgesetzen die soziale Integration der Inhaftierten als spezialpräventives Vollzugsziel fest, wenn sie als Ziel die Befähigung der Gefangenen, „künftig in sozialer Verantwortung ein Leben ohne Straftaten zu führen "4, betonen. Der Jugendstrafvollzug ist motivierender Erziehungsvollzug und bietet den Jugendlichen und Heranwachsenden zahlreiche Möglichkeiten zur Re- (besser: Nach- $)^{5}$ Sozialisierung. Auf der anderen Seite scheint mit einer Rückfallquote von $78 \%$ die Verwertung bzw. die Verwertbarkeit der angebotenen Chancen jedoch gering: Mehr als drei Viertel der entlassenen Jugendstrafgefangenen werden wieder straffällig, hier im Sinne von zumindest einer erneuten Eintragung im Bundeszentralregister im Zeitraum von vier Jahren nach der Entlassung. ${ }^{6}$ Sie konnten offensichtlich die im Vollzug angebotenen Chancen zur nachholenden Gesamterziehung nicht nutzen oder nach der Entlassung in Freiheit adäquat verwerten und bleiben von der Gesellschaft ausgegrenzt. Auf diese Exklusion weist auch das Thema des 27. Deutschen Jugendgerichtstages 2007 „Fördern, Fordern, Fallen lassen“ hin. ${ }^{7}$

1 Achternbusch 1986, 229.

2 Vgl. hierzu Ostendorf 2007, 111; Walter 2011, 99 ff.; Dünkel / Pörksen, NK 2/2007, 64; Walkenhorst, APuZ 7/2010, 26.

3 BVerfGE 116, 69ff. (2 BvR 1673/04 vom 31.05.2006).

4 Vgl. hierzu $\$ 2$ LJVollzG RLP.

$5 \mathrm{Zu}$ dieser Begriffskorrektur vgl. Walkenhorst 2002, 322.

6 Jeble u. a. 2010, 55.

7 Sonnen, NK 2/2007, 50.

NK 25. Jg. 3/2013 
Seit der Etablierung der pädagogischen Unterweisung in den Haftanstalten Mitte des 19. Jahrhunderts in Deutschland ${ }^{8}$ wird den jugendlichen Inhaftierten die Chance geboten, ihre Bildungslücken während des Vollzuges aufzuarbeiten und sich weiterzubilden, da Bildungsdefizite und sittliche Verwahrlosung als ursächlich für deren delinquentes Verhalten angesehen werden. ${ }^{9}$

Mittlerweile hat sich ein Paradigmenwechsel vollzogen: Der jugendliche Delinquent wird mit seinen Defiziten und Schwächen weniger als Objekt korrigierender Erziehung während der Haft gesehen, sondern eher als Subjekt und Mitwirkender im vollzuglichen Behandlungsprozess betrachtet, der bei seinen Begabungen und Stärken abzuholen ist, um ihm über deren Ausbau eine Chance zur Resozialisierung zu geben. „Leitbild ist nicht mehr der defizitäre, sondern der entwicklungsfähige junge Mensch. " ${ }^{10} \mathrm{Im}$ Rahmen dieser nachholenden Gesamterziehung haben schulische Bildungsmaßnahmen im Jugendvollzug seit jeher einen festen Platz. Jedoch müssen bereits nicht delinquente Schüler an öffentlichen (Haupt-) Schulen in Freiheit um den Erhalt eines adäquaten Arbeitsplatzes, der ihnen einen angemessenen sozioökonomischen Status sichert, kämpfen. ${ }^{11}$ Viele Schüler, die (mühsam) eine Grundbildung in Form des Hauptschulabschlusses erworben haben, resignieren und werden zu einem KoZ $=$ Kind ohne $\mathrm{Zukunft}^{12}$; in der Jugendsprache hat sich der Begriff „Honk“ = Hauptschüler ohne nennenswerte Kenntnisse etabliert. Der Hauptschule hängt der Ruf einer Restschule an. Entlassene Jugendstrafgefangene sind noch zusätzlich mit dem Makel der Vorstrafe beschwert. Stuft die PISA-Studie Schüler mit defizitären Kenntnissen (nämlich die, die die PISA-Kompetenzstufe I nicht erreicht haben) im Hinblick auf ihre späteren beruflichen Erfolgsaussichten als Risikoschüler ein ${ }^{13}$, so potenziert sich diese Risikoeinstufung bei Jugendstrafgefangenen durch die zusätzliche Stigmatisierung als Kriminelle.

Die Schule im Jugendstrafvollzug umfasst freizeitbezogene Angebote, den Unterricht zum Erwerb von elementaren Kenntnissen und Fertigkeiten mit lebenspraktischem Bezug (Alphabetisierung, Elementar- und Vorbereitungskurse), die Vermittlung einer allgemeinen, berufsorientierten Grundbildung (Hauptschulabschluss) sowie weiterführende mittlere Bildungsabschlüsse (Mittlere Reife), um die Chance der Jugendlichen auf ein künftiges straffreies Leben zu erhöhen. Gemäß den in den Justizvollzugsgesetzen verankerten Grundsätzen der Vollzugsgestaltung ist „das Leben im Vollzug den allgemeinen Lebensverhältnissen so weit wie möglich anzugleichen. " 14 Dies zeigt sich auch daran, dass sich der schulpolitische Pluralismus in den Vollzugsschulen der Länder widerspiegelt. Fraglich ist nun, ob die Schule im Jugendvollzug tatsächlich auf dem aktuellen Stand ist oder ob sie - wie Myschker vermutet - „der Entwicklung im übrigen Schulwesen

8 Vgl. hierzu Wichern 1973, 179 f.; Krohne 1889, 500; Liszt 1905, 397.

9 Vgl. hierzu auch Feltes, WPB 3/1979, 98.

10 Walter 2011, 101.

11 Vgl. hierzu Autorengruppe Bildungsberichterstattung 2010, 99.

12 Preisendörfer 2008, 51.

13 Baumert 2001, $116 \mathrm{ff}$.

14 Vgl. $\$ 7$ Abs. 1 LJVollzG RLP. 
hinterherhinkt ${ }^{\text {“15 }}$ und, auch in Anbetracht der oben dargestellten Entwertung des Hauptschulabschlusses, eine Nachjustierung erfolgen muss. Bei den folgenden Überlegungen stehen die abschlussbezogenen schulischen Bildungsmaßnahmen im Fokus der Betrachtung, weil bei dem für die Legalbewährung bedeutsamen späteren Übergang ins Berufsleben großer Wert auf formelle schulische Qualifikationen gelegt wird. Zudem beschränkt sich die beabsichtigte Analyse der Ausrichtung schulischer Bildungsmaßnahmen auf die Jugendstrafhaft: Die Untersuchungshaft bleibt unberücksichtigt, da schulische Bildungsmaßnahmen speziell für junge Untersuchungsgefangene einer hohen Teilnehmerfluktuation und dem Umstand unterliegen, dass sich viele junge Untersuchungsgefangene in der Hoffnung auf eine baldige Entlassung nicht auf langfristige Bildungsmaßnahmen im Vollzug einlassen wollen. ${ }^{16}$

\section{B. Ausrichtung der Schule im Jugendstrafvollzug}

\section{Vorbildungsstand bei Haftantritt}

Berichterstattungen in den Medien vermitteln oft den Eindruck, der Jugendknast sei ein Hort der Einfältigkeit und Gewalt. ${ }^{17}$ Zum Vorbildungsstand der Jugendstrafgefangenen bei Inhaftierung finden sich im Internet Äußerungen wie „ein hoher Prozentsatz der Inhaftierten verfügt bei Haftantritt weder über einen Schulabschluss noch über eine Berufsausbildung." 18 Auch das Bundesverfassungsgericht legt in seinem Urteil vom 31.05.2006 zugrunde, dass „der weitaus größte Teil der Gefangenen über keinerlei Schuloder Ausbildungsabschluss verfüge. " ${ }^{19}$ Hierzu besteht unter den meisten Vollzugspraktikern Einigkeit darüber, dass diese Bildungsdefizite nicht Ausdruck einer genuinen Intelligenzschwäche sind, sondern als das Resultat von Misserfolgsängstlichkeit, mangelndem Durchhaltevermögen, Schulunlust und Schulverweigerung in der sekundären Sozialisationsphase zu betrachten sind. Hans-Jürgen Eberle berichtet nach Untersuchung der Intelligenz- und Schulleistungswerte von 142 jungen Untersuchungsgefangenen in den Jahren 1973/74: „Das Leistungspotential der Probanden liegt deutlich über der tatsächlich erbrachten Schulleistung. Von der Leistungsmöglichkeit her entspricht die Probandengruppe der Gesamtbevölkerung [Unterstreichung im Original, d. Verf.].“20

Wenn der Jugendvollzug im Rahmen der tertiären Spezialprävention diese Leistungspotenziale und schlummernden Ressourcen der Jugendlichen und Heranwachsenden effektiv und nachhaltig nutzen will, müssen die Inhaftierten mit ihren Schwächen und

15 Myschker 2009, 21.

16 Zum qualifizierenden Hauptschulabschluss in der U-Haft vgl. Lang, ZfStrVo 3/2001, 152 ff.

17 Zum Anstieg der gewaltaffinen Tätergruppen im Jugendvollzug vgl. Walkenhorst, APuZ 7/2010, 23.

18 Oblendorf, Schulabschluss im Gefängnis, Bericht über die JSA Regis-Breitingen (zuletzt besucht am 15.06.2013).

19 BVerfGE 116, 69, 78 (2 BvR 1673/04 vom 31.05.2006).

20 Eberle 1980, 296; vgl. auch Hoffmann 1989, 467; Myschker / Hoffmann 1984, 41; Schmid, Zeitschrift für Strafvollzug: für Praxis und Wissenschaft 6/1964, 336.

NK 25. Jg. 3/2013 
Problemen dort abgeholt werden, wo sie zu Beginn der Haft stehen. ${ }^{21}$ So kann auf ihren Fähigkeiten aufgebaut werden. Gemäß mehreren Evaluationsstudien ${ }^{22}$ zum Jugendvollzug kommt abschlussbezogenen schulischen Bildungsmaßnahmen eine besondere Bedeutung zu, da sie im Hinblick auf die (Wieder-)Eingliederung positive Effekte zeigen, vor allem wenn sich berufliche Bildungsmaßnahmen daran anschließen. Dabei ist neben dem Faktor der zur Verfügung stehenden Haftzeit vor allem der Vorbildungsstand bei Haftantritt wichtig. Bundesweite Erhebungen, die sich auf einen bestimmten Stichtag beziehen müssten, liegen hierzu nicht vor. ${ }^{23}$ Eine lokale Erhebung von 185 Jugendstrafgefangenen im Juli/August 2011 aus der rheinland-pfälzischen Jugendstrafanstalt Schifferstadt ergab folgendes Bild:

\section{Vorbildung der Jugendlichen und Heranwachsenden}

bei Strafhaftantritt in der Jugendstrafanstalt Schifferstadt; Stand: Juli / August 2011 Gesamtzahl: $\mathrm{n}=185$

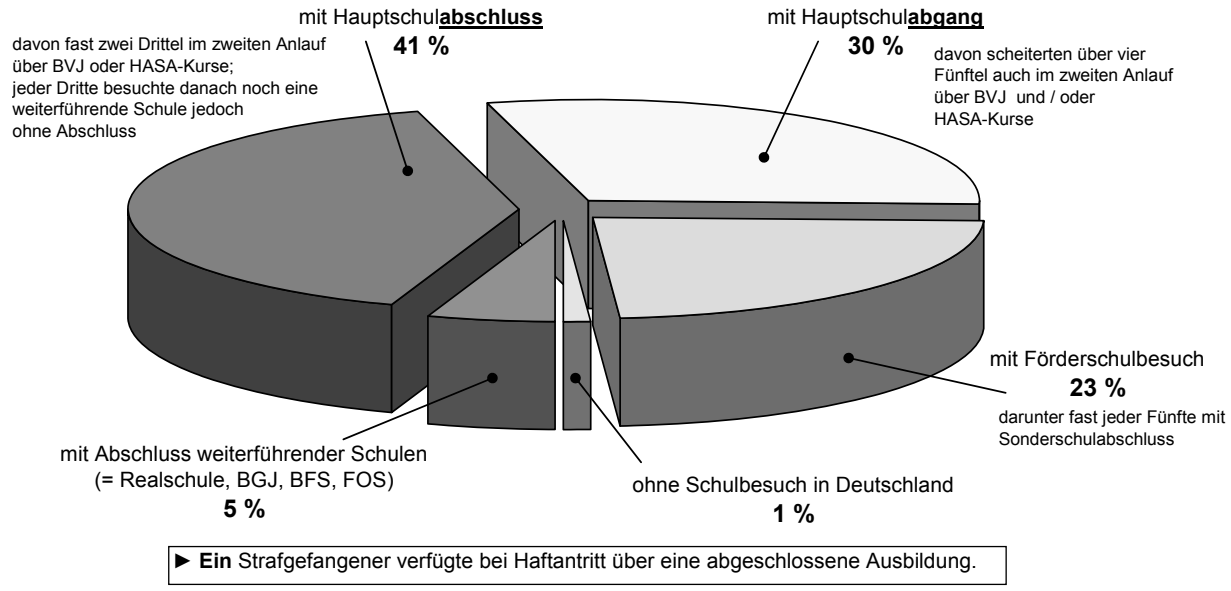

Hiernach besaßen bei Strafhaftantritt ca. 46\% der Jugendlichen und Heranwachsenden zumindest den Hauptschulabschluss; nur ein Gefangener verfügte über eine abgeschlossene Berufsausbildung, als er inhaftiert wurde. Es zeigt sich eine Überrepräsentanz der

21 Vgl. Walkenhorst 2002, 342.

22 Vgl. hierzu Berckhauer / Hasenpusch 1982, 322; Dolde / Grübl 1996, 284; Giebel, „Rückfälligkeit" bei jugendlichen Straftätern in den Jahrgängen Ende 1996 - Anfang 2000 (zuletzt besucht am 15.06.2013), Baumann, ZfStrVo 1/1984, 36; Wirth, Zeitschrift für soziale Strafrechtspflege 44/2007, 16.

23 Im April 2012 hat Frau Dipl.-Päd. Susann Reinheckel ihre Doktorarbeit „Geringqualifikation bei männlichen Strafgefangenen im geschlossenen Jugendstrafvollzug der Bundesrepublik Deutschland - eine empirische Untersuchung " an der Humboldt-Universität zu Berlin eingereicht, die auch eine bundesweite Totalerhebung zum schulischen Angebot in den Jugendanstalten der BRD umfasst. Sie wurde mittlerweile veröffentlicht und liefert zum Vorbildungsstand bei Haftantritt ähnliche Ergebnisse, vgl. Reinheckel 2013, 135f. (zuletzt besucht am 20.08.2013). 
ehemaligen Förder- und Hauptschüler im Vollzug gegenüber deren Anteil in den Schulen außerhalb der Mauern: Besuchten in Rheinland-Pfalz im Schuljahr 2010/11 3,3\% der Schüler eine Förderschule, beträgt der Anteil ehemaliger Förderschüler in der Jugendstrafanstalt Schifferstadt 23\%, bei den Hauptschülern ist das Verhältnis $71 \%$ Inhaftierte mit vorherigem Hauptschulbesuch zu 7,1\% Hauptschüleranteil an den öffentlichen Schulen, wobei bei diesen Angaben auf das Schuljahr 2008/09 zurückgegriffen werden muss, da ab dem Schuljahr 2009/10 viele Hauptschulen in die Realschule plus überführt wurden. ${ }^{24}$

Die Jugendstrafanstalt Schifferstadt ist eine vor über 20 Jahren in Dienst gestellte Anstalt des geschlossenen, behandlungsorientierten Wohngruppenvollzuges mit $234 \mathrm{Haft}$ plätzen (182 Strafhaft, 52 Plätze U-Haft) und nach dem Vollstreckungsplan bezüglich der Strafhaft für den Vollzug von Jugendstrafen aus den Landgerichtsbezirken im südlichen Rheinland-Pfalz zuständig. Da es sich um eine regionale Zuständigkeit handelt und nicht wie bei einigen anderen Jugendanstalten die Zuweisung nach Alter oder Delikten erfolgt, kann von einer Repräsentativität der Ergebnisse hinsichtlich des Vorbildungsstandes ausgegangen werden. Auch die weiteren Strukturdaten der Jugendstrafanstalt Schifferstadt, wie Alters- und Deliktstruktur, Ausländeranteil und Aufenthaltsdauer im Vollzug entsprechen denen des bundesdeutschen Jugendstrafvollzuges. Es kann somit vorausgesetzt werden, dass die bewusste Auswahl der Stichprobe in Form der zu einem bestimmten Zeitpunkt in der Jugendstrafanstalt Schifferstadt inhaftierten Gefangenen „ein verkleinertes Abbild der Grundgesamtheit [hier aller Jugendstrafgefangenen in Deutschland, d. Verf.] darstellt und somit eben die Grundgesamtheit ,repräsentiert'. "25 Unterstellt man die Repräsentativität des erhobenen Vorbildungsstandes, so bedeutet dies, dass nur etwa die Hälfte der Jugendstrafgefangenen $(=54 \%)$ ihre Haft ohne Hauptschulabschluss antrat. Damit treffen die obigen Aussagen, dass „der weitaus größte Teil der Jugendstrafgefangenen über keinerlei Schulabschluss verfüge “26 nicht mehr zu und dies aus dem Grunde, weil sich nach Ansicht des Verfassers der Vorbildungsstand in den letzten Jahrzehnten im Jugendstrafvollzug in Richtung mehr Hauptschulabschlüsse verschoben hat. Im Folgenden soll die Nullhypothese überprüft werden, die Wolfgang Wirth in seinem Beitrag in der Zeitschrift Forum Strafvollzug liefert: „Die Qualifizierungsdaten [gemeint ist das schulische und berufliche Bildungsniveau, d. Verf.]

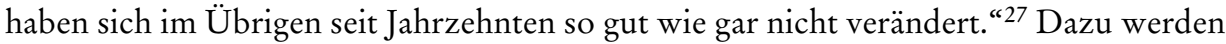
zwei Untersuchungen durchgeführt. Zuerst erfolgt im Rahmen einer quantitativen Längsschnittuntersuchung ein Vergleich der Daten zum Vorbildungsstand der Jugendstrafgefangenen in Schifferstadt über den Zeitraum 1999 bis 2011. Danach wird untersucht, ob sich aus der vorhandenen Literatur eine Tendenz zur Änderung der Vorbildung im Jugendstrafvollzug erkennen lässt.

24 Die genannten Zahlen zu den Schüleranteilen in Rheinland-Pfalz stammen aus: Statistisches Landesamt Rheinland-Pfalz, Allgemeinbildende Schulen im Schuljahr 2010/2011, 9 f. (zuletzt besucht am 15.06.2013.).

25 Diekmann 2010, 391.

26 BVerfGE 116, 69, 78 (2 BvR 1673/04 vom 31.05.2006).

27 Wirth, FS 2/2009, 76; vgl. auch Reinheckel 2013, 186.

NK 25. Jg. 3/2013 


\section{Untersuchungen zur Änderung des Vorbildungsstandes}

1. Veränderung des Vorbildungsstandes in der Jugendstrafanstalt Schifferstadt von 1999 bis 2011

Um den Vorbildungsstand der Jugendstrafgefangenen im Längsschnitt der Jahre zu betrachten, wurde aus den Vollzugsplänen der bei Haftantritt erreichte höchste Schulabschluss erfasst und ausgezählt. Es wurden dabei alle Strafgefangenen berücksichtigt, die sich zum Schuljahresbeginn in den Spätsommermonaten 1999, 2001, 2005, 2007, 2009 und 2011 in der Jugendstrafanstalt Schifferstadt befanden. Leider wurden für das Jahr 2003 keine Daten erhoben.

Zur Gewährleistung einer Vergleichbarkeit der Stichproben wurde darauf geachtet, dass die Auszählungen jeweils in den gleichen Monaten erfolgten, da sich in den Jugendstrafanstalten die Gefangenenpopulationen je nach Jahreszeit unterscheiden können, z. B. werden nach Schuljahresende viele Gefangene entlassen, die nach Entlassung eine Schul- oder Berufsausbildung beginnen wollen und damit eine günstige Prognose aufweisen. Um Doppelzählungen zu vermeiden, wurde der bei Haftantritt höchste vorhandene Schulabschluss berücksichtigt. In dieser Weise wurden auch Jugendliche gewertet, die aus einer laufenden Schulmaßnahme verhaftet wurden. Solche Fälle treten im Übrigen nur selten auf, denn die meisten jugendlichen Delinquenten sind sich darüber im Klaren, dass sie wegen ihrer Taten demnächst eine Haftstrafe antreten müssen, und hatten deshalb bereits frühzeitig einen Schulbesuch beendet. Doppelt wurden jedoch die (wenigen) Jugendstrafgefangenen mit Berufsabschluss berücksichtigt, da sie bereits in die Zählung der Schulabschlüsse eingingen.

Aus dieser Trendstudie zum Vorbildungsstand in den Jahren 1999, 2001, 2005, 2007, 2009 und 2011 der Jugendstrafgefangenen in der Jugendstrafanstalt Schifferstadt ergibt sich, dass der Anteil der Inhaftierten, die bei Haftantritt über einen Hauptschulabschluss oder den Abschluss einer weiterführenden Schule (10. Klasse Hauptschule, Integrierte Gesamtschule, Realschule, Berufsgrundschuljahr, Berufsfachschule I/II, Fachoberschule, Gymnasium ab 10. Klasse aufwärts) verfügen, stetig gestiegen ist, und zwar von $20 \%$ im Jahr 1999 auf nunmehr 46\% im Jahr 2011. Im Gegenzug sind die Anteile der Jugendstrafgefangenen, deren Bildungsstand bei Inhaftierung nicht dem Hauptschulabschluss entsprach (ohne Schulbesuch in Deutschland und kein Schulabschluss, Förderschulbesuch, Abgang aus der Hauptschule), stetig gesunken (1999: 80\%; 2011: 54\%). Seit 2007 wurde für diese Gruppe auch erfasst, wer anschließend in Freiheit noch - allerdings erfolglos - versucht hatte, quasi in einem „zweiten“ (manchmal auch dritten) „Anlauf“ den Hauptschulabschluss im sogenannten Übergangssystem ${ }^{28}$ zu erwerben, wer also ein Berufsvorbereitungsjahr an der Berufsschule oder einen Hauptschulabschlusskurs bei einem freien Träger besucht hatte. Der Anteil dieser auch im Übergangssystem erfolglosen Schüler ist von 61\% im Jahr 2007 auf 71\% im Jahr 2011 gestiegen. Der Prozentsatz der Jugendstrafgefangenen, die bei Haftantritt bereits auf eine erfolgreich abgeschlossene 
Berufsausbildung zurückblicken konnten, verharrt in dem 13-Jahreszeitraum auf konstant niedrigem Niveau (1 bis maximal 5 Gefangene, entsprechend 0,5 bis 2,7\%). Die Entwicklung der Gefangenengesamtzahlen (n) entspricht dem bundesdeutschen Trend. Erheblich gesunken ist der Anteil derjenigen Gefangenen, die in Deutschland nie regelmäßig die Schule besucht hatten und bei Strafantritt auch über keinen Schulabschluss verfügten (1999: 12\%; 2011: 1\%).

Veränderung des Vorbildungsstandes bezogen auf den Hauptschulabschluss bei Jugendstrafgefangenen in der Jugendstrafanstalt Schifferstadt jeweils zu Schuljabresbeginn 1999 - 2011

\begin{tabular}{|c|c|c|c|c|}
\hline Jahr & $\begin{array}{l}\text { bei Inhaftierung: } \\
\text { kein Hauptschulab- } \\
\text { schluss }\end{array}$ & $\begin{array}{l}\text { bei Inhaftierung } \\
\text { mindestens Haupt- } \\
\text { schulabschluss }\end{array}$ & $\begin{array}{l}\text { Gesamt } \\
\text { n }\end{array}$ & $\begin{array}{l}\text { mit abgeschlos- } \\
\text { sener Ausbil- } \\
\text { dung }\end{array}$ \\
\hline 1999 & $187=80,0 \%$ & $46=20,0 \%$ & 233 & $5=2,1 \%$ \\
\hline 2001 & $208=70,7 \%$ & $86=29,3 \%$ & 294 & $5=1,7 \%$ \\
\hline 2003 & & & & \\
\hline 2005 & $164=67,2 \%$ & $80=32,8 \%$ & 244 & $3=1,2 \%$ \\
\hline 2007 & $118=62,1 \%$ & $72=37,9 \%$ & 190 & $3=1,6 \%$ \\
\hline 2009 & $100=53,8 \%$ & $86=46,2 \%$ & 186 & $5=2,7 \%$ \\
\hline 2011 & $100=54,1 \%$ & $85=45,9 \%$ & 185 & $1=0,5 \%$ \\
\hline
\end{tabular}

Übersicht zur Veränderung des Vorbildungsstandes mit kumulierten Werten

Bezüglich der Entwicklung des Vorbildungsstandes der Inhaftierten in der Jugendstrafanstalt Schifferstadt über die Jahre 1999 - 2011 ergibt sich:

Das Streudiagramm zeigt eine lineare Regression mit dem Determinationskoeffizienten $\mathrm{R}^{2}=0,9426$, was die Korrelation zwischen Untersuchungsjahr und dem Anteil der Gefangenen mit mindestens Hauptschulabschluss (bzw. denjenigen ohne Hauptschulabschluss) bestätigt (Produkt-Moment-Korrelationskoeffizient $\mathrm{R}=0,9708$ ). Bei Zu- 
grundelegung eines Signifikanzniveaus von $\alpha=0,05$ ergibt sich ein $\mathrm{p}$-Wert $<0,0126 .{ }^{29}$ Im Rahmen dieser linearen Regressionsanalyse ist das Ergebnis also als signifikant und nicht als zufallsbedingt zu betrachten. Die Nullhypothese ist zu verwerfen und die Alternativhypothese bestätigt:

In den letzten 13 Jabren hat sich der Vorbildungsstand geändert und zwar insofern, dass die Zabl der Jugendstrafgefangenen, die bei Inhaftierung bereits über mindestens einen Hauptschulabschluss verfügten, zumindest in der Jugendstrafanstalt Schifferstadt deutlich gestiegen ist. Im Gegenzug sank die Zahl der Jugendstrafgefangenen, die bei Haftantritt keinen Hauptschulabschluss besaßen.

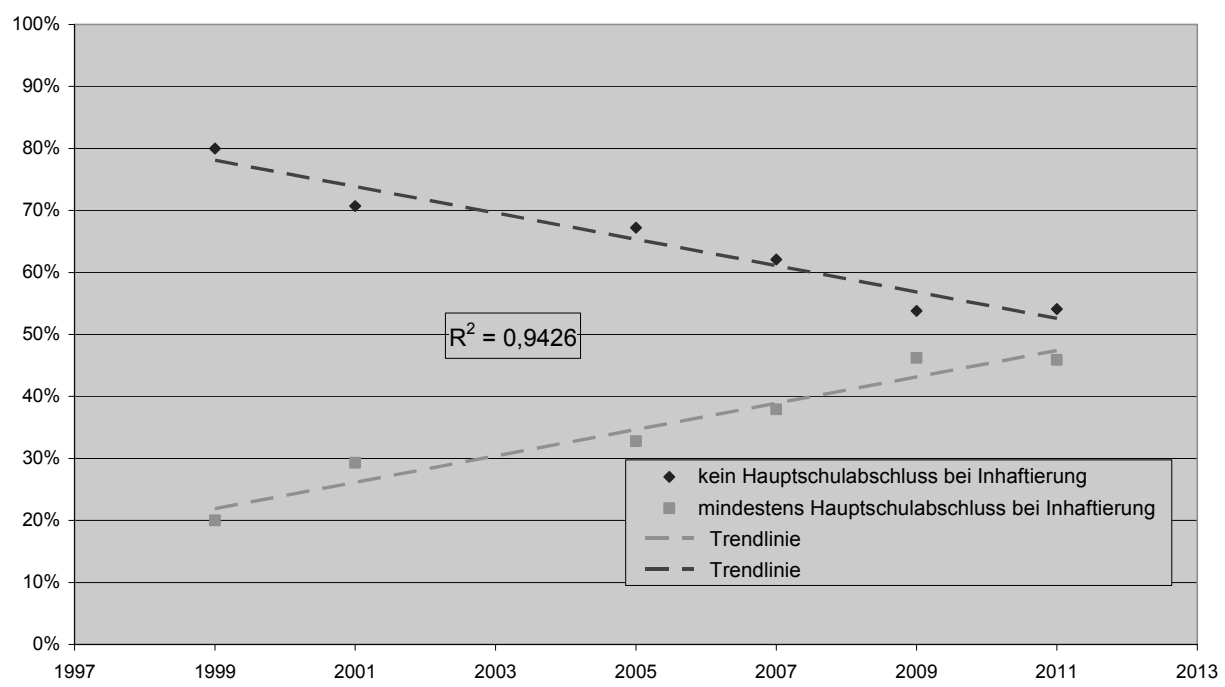

Streudiagramm zur Veränderung des Vorbildungsstandes

Die Berechnung des Determinationskoeffizienten für die Gefangenen, die die Haft bereits mit einem abgeschlossenen Beruf antraten, ergibt $R^{2}=0,1056(p<0,5297)$ und bedeutet somit, dass die Verteilung eher zufällig ist und kein Trend hinsichtlich des Vorliegens eines Berufsabschlusses bei Haftantritt erkannt werden kann, was bei den geringen Fallzahlen ohnehin kaum möglich ist.

2. Veränderung des Vorbildungsstandes anhand der wissenschaftlichen Literatur

In der wissenschaftlichen Literatur finden sich einige Quellen, die ebenfalls über den Vorbildungsstand von (Jugend-) Strafgefangenen bei Haftantritt berichten. Die dort gemachten Angaben zur schulischen Bildung werden verglichen und dahingehend überprüft, ob sich auch hier eine Tendenz zu einem höheren Anteil Jugendstrafgefangener,

29 Die Berechnung der Werte erfolgte mit der Software Statistica 9.1. 
die bei Haftantritt mindestens einen Hauptschulabschluss besaßen, erkennen lässt. Bei diesem Vergleich ist zu beachten, dass sich zum einen die in den jeweiligen Querschnittserhebungen betrachteten Gefangenenpopulationen beispielsweise hinsichtlich Haftart (Einbeziehung der U-Haft, des offenen Vollzuges), Nationalität und Alter (je nach Vollstreckungsplan sind einige Anstalten nur für bestimmte Altersgruppen zuständig) unterscheiden können. Zum anderen wurden bei der Auszählung des Vorbildungsstandes von den jeweiligen Untersuchern unterschiedliche Kriterien (Problem der Mehrfachzählung erreichter Schulabschlüsse) zugrunde gelegt.

- Johann Hinrich Wichern, der sich für die Etablierung des Schulunterrichts in einem jugendgemäßen Vollzug einsetzte, stufte 1860 die Vorbildung der Gefangenen als desaströs ein, wenn er von „einem so hohen, dieser Unwissenheit entsprechenden Grad von Stumpfsinn " 30 spricht. Karl Krohne, der im Unterricht eher eine ungerechtfertigte Vergünstigung während der Haft sah, wird genauer: „Unter 6677 Männern und 1420 Weibern, welche 1886/87 in den preußischen Strafanstalten in Zugang kamen, hatten nur 60 Männer oder 0,9 Proz. eine höhere als Elementarbildung; 1712 Männer und 253 Weiber oder 25 Proz., bzw. 17 Proz. Elementarbildung; [...].“31

- Hammermann, 1973, JVA Staumühle / NRW, 24,9\% der Jugendstrafgefangenen mit mindestens Volksschulabschluss. ${ }^{32}$

- Großkelwing, 1975, Niedersachsen, $\underline{38 \%}$ der Jugendstrafgefangenen mit Schulabschluss (inkl. Sonderschulabschluss). ${ }^{33}$

- Hans-Joachim Kruse, Jugendvollzugsanstalt Bremen-Blockland:

a) 1976: 20,6\% mit Hauptschul- oder Realschulabschluss.

b) 1986: $27,3 \%$ mit mindestens Hauptschulabschluss. ${ }^{34}$

- Hilkenbach, 1976/77, JSA Herford, 29,8\% mit mindestens Hauptschulabschluss. ${ }^{35}$

- Myschker/Hoffmann, 1983, Hamburg, $\underline{36 \%}$ der Jugendstrafgefangenen mit mindestens Hauptschulabschluss. ${ }^{36}$

- Matt / Maul, 2003, Bremen, $\underline{34 \%}$ mit mindestens Hauptschulabschluss. ${ }^{37}$

- Internetportal ZUM-Wiki, 2003, Bayern, $46 \%$ mit Hauptschulabschluss. ${ }^{38}$

- Zelinka, 2001/2002, JVA Ottweiler / Saarland, $\underline{50,4 \%}$ der Jugendstrafgefangenen mit mindestens Hauptschulabschluss. ${ }^{39}$

- Elfriede Sessar-Karpp nennt für 1976 bei 175 Jugendstrafgefangenen aus Baden-Württemberg (JVA Adelsheim) einen Anteil von 33,7\% der Inhaftierten, die mindestens den Hauptschulabschluss erreicht hatten (in dieser Statistik besteht jedoch das Pro-

30 Wichern 1973, 179.

31 Krobne 1889, 479.

32 Hammermann, ZfStrVo 1977, 137.

33 Großkelwing 1976, 297.

34 Kruse 1999, $86 \mathrm{f}$.

35 Hilkenbach, ZfStrVo 2/1979, 83.

36 Myschker / Hoffmann 1984, 37.

37 Matt / Maul, ZfStrVo 4/2005, 198.

38 ZUM-Wiki, Schule in der JVA (zuletzt besucht am 15.06.2013).

39 Zelinka 2010, $26 \mathrm{f}$.

NK 25. Jg. 3/2013 
blem der Mehrfachberücksichtigung bei den erreichten Schulabschlüssen). Außerdem merkt Frau Sessar-Karpp an, dass „,in der Testanstalt der Anteil der Inhaftierten mit Hauptschulabschluß in den letzten Jahren ab-, und der der Sonderschüler zuzunehmen scheint. “40

- Gabriele Dolde und Günter Grübl kommen in einer anderen Erhebung an 401 deutschen Jugendstrafgefangenen der JVA Adelsheim in den Jahren 1976/77 zu dem Ergebnis, dass 46,7\% der Inhaftierten zuvor die Sonder- bzw. Hauptschule mit Abschluss oder eine weiterführende Schule besuchten. Hiervon abzuziehen wären noch die Sonderschüler mit Abschluss, und es blieben die (damals wenigen) ausländischen Gefangenen unberücksichtigt, so dass sich diese Ergebnisse den oben genannten von Frau Sessar-Karpp aus dem ähnlichen Zeitraum annähern. Dolde / Grübl merken zur Entwicklung des Vorbildungsstandes an, dass „in den 80er Jahren der formale Bildungsstand der Jugendstrafgefangenen etwas gestiegen ist: 1991 hatten rund 60\% der Zugänge mindestens den Hauptschulabschluß.“41

- Wolfgang Stelly und Jürgen Thomas, beide vom Institut für Kriminologie der Universität Tübingen, geben für 420 Jugendstrafgefangene der JVA Adelsheim für 2009/2010 an, dass 50\% der Inhaftierten bei Haftantritt einen Hauptschulabschluss besaßen und dass $\underline{5 \%}$ der Jugendstrafgefangenen über einen höheren Abschluss als den Hauptschulabschluss verfügten. Stelly und Thomas vergleichen diesen Anteil mit einer repräsentativen Jugendstudie (27\% mit höherer Schulbildung im DJI-Jugendsurvey) und vermuten, dass „bei den Jugendstrafgefangenen von einzelnen Ausnahmen abgesehen maximal eine weitere Erhöhung des Hauptschulabschlussanteils zu erwarten ist. “42

Auch unter Beachtung der oben gemachten Einschränkungen zur Aussagefähigkeit dieser vergleichenden Betrachtung ist aus diesen Quellen in der wissenschaftlichen Literatur eine Verschiebung des Vorbildungsstandes in Richtung Hauptschulabschlussniveau bei Antritt der Jugendstrafe erkennbar. Berichteten Wichern und Krobne Mitte bzw. Ende des 19. Jahrhunderts noch von „stumpfsinnigen“ Gefangenen, besaß in den 70er Jahren ungefähr ein Viertel der Jugendstrafgefangenen bei Haftantritt den Hauptschulabschluss, während heute annähernd jeder zweite Jugendstrafgefangene bei Haftbeginn einen Hauptschulabschluss nachweisen kann, was auch die eigene Erhebung aus der Jugendstrafanstalt Schifferstadt belegt. Schien es so, dass in den 70er Jahren nach der obigen Anmerkung von Frau Sessar-Karpp immer weniger Jugendstrafgefangene mit Berufsreife ihre Haft antraten, hat sich dieser Trend in den letzten Jahren umgekehrt, worauf auch die Beobachtungen von Dolde / Grübl und Stelly / Thomas hindeuten: Der Anteil der Jugendstrafgefangenen, die mit einem (formal) besseren Bildungsstand ihre Jugendstrafe antreten, ist gestiegen.

40 Sessar-Karpp 1982, 149.

41 Dolde / Grübl 1996, 240.

42 Stelly/Thomas 2011, 140.

TITEL 


\section{Diskussion und Schlussfolgerungen}

Als ursächlich für den (zumindest formell) gestiegenen Vorbildungsstand der Jugendstrafgefangenen in Form des erhöhten Anteils jugendlicher Delinquenten, die bei Haftantritt bereits über den Hauptschulabschluss verfügen, sind folgende Punkte zu betrachten:

- Der formelle schulische Bildungsstand der Schulentlassenen hat sich in den letzten 20 Jahren erheblich verbessert: Verließen 1992 noch 8,2\% die Schule ohne Hauptschulabschluss, waren es 2006 nur 7,8\% und im Jahr 2011 sogar nur 5,6\% der Schulentlassenen (Zahlen des Statistischen Bundesamtes für das Schuljahr 2011/1243).

- Der prozentuale Anstieg der Jugendlichen, die zumindest über eine allgemeine Basisbildung in Form des Hauptschulabschlusses verfügen, ist auf den Ausbau des Übergangssystems zurückzuführen, in dessen Bildungsmaßnahmen viele Jugendliche an eine allgemeine Berufsreife herangeführt werden konnten. Immerhin durchlaufen mehr als drei Viertel der Schüler ohne Hauptschulabschluss das Übergangssystem, wo es dann noch einmal gelingt, „die Quote der Jugendlichen ohne Schulabschluss tendenziell zu halbieren.“44

- Ein Abschlusszeugnis der Hauptschule, die 1964 aus der Oberstufe der Volksschule als Reaktion auf den Sputnik-Schock und die vermeintliche bildungspolitische Vormachtstellung der UdSSR entstand, ist am Ausbildungsmarkt nahezu wertlos. 1994 hält Hans-Joachim Kruse im Zusammenhang mit der schulischen Qualifizierung Jugendstrafgefangener fest: „Der Hauptschulabschluß stellt den untersten qualifizierten Level unseres Bildungssystems dar. Unterhalb dieses Standards ist praktisch von Nichtbildung zu sprechen. " ${ }^{45}$ Diese Äußerung ist heute nach fast 20 Jahren als sehr optimistisch einzustufen, wenn das Jahresgutachten 2007 des Aktionsrates Bildung feststellt: „Über Jahrzehnte hinweg war das Erreichen einer Berufsausbildung für Schulabgänger ohne oder mit niedrigem Schulabschluss die Regel. Mit Beginn der Bildungsexpansion in den 1970er Jahren begann allerdings ein Verdrängungsprozess zum Nachteil der Schulabgänger mit niedrigem oder gar keinem Abschluss. Stattdessen drängten zunehmend auch Absolventen mit Realschulabschluss/Mittlerer Reife und mit Hochschulreife in den Ausbildungsmarkt. Zugleich veränderte sich die Wertigkeit von Hauptschulabschlüssen negativ, die Hauptschule gilt seither als ,Restschule'. Dementsprechend wandelte sich auch das Auswahlverhalten der Betriebe. Hauptschulabgänger bzw. Jugendliche ohne Schulabschluss stehen in Konkurrenz zu Mitbewerbern mit höherem Schulabschluss, wodurch sich ihre Chancen, ins duale Berufsausbildungssystem einzumünden, erheblich verschlechtern. Damit verbunden

43 Statistisches Bundesamt, Fachserie 11, Reihe 1. Bildung und Kultur. Allgemeinbildende Schulen Schuljahr 2011/2012, 278 (zuletzt besucht am 15.06.2013); vgl. hierzu auch Klemm, Jugendliche ohne Hauptschulabschluss, 13 (zuletzt besucht am 15.06.2013); Autorengruppe Bildungsberichterstattung 2010, 90.

44 Klemm, Jugendliche ohne Hauptschulabschluss, 25 (zuletzt besucht am 15.06.2013).

45 Kruse, Unsere Jugend 5/1994, 194.

NK 25. Jg. 3/2013 
stehen diesen Jugendlichen immer weniger Berufe zur Auswahl.“46 Der Schriftsteller Bruno Preisendörfer fasst diese Exklusion der Hauptschüler kritisch zusammen: „Das Konkurrenzdefizit der Hauptschule macht jeden ihrer Schüler zu einem KoZ: Kind ohne Zukunft - keine Ausbildung, kein Job“47 und zeichnet die Entwertung der Hauptschule mit der Reihung „Volksschule. Hauptschule. Restschule. Rütlischu$1 e^{“ 48}$ nach. In Abwandlung der oben zitierten Äußerung von Hans-Joachim Kruse ist schon beim Hauptschulabschluss im Hinblick auf dessen Verwertbarkeit am Arbeitsmarkt heute quasi von Nichtbildung, zumindest von unzureichender Minimalbildung $\mathrm{zu}$ sprechen.

Die drei genannten schulstrukturellen und bildungspolitischen Veränderungen haben dazu geführt, dass auch im Jugendstrafvollzug der Anteil der Inhaftierten mit Hauptschulabschluss in den letzten Jahren gestiegen ist, während die Zahl der Inhaftierten, die bei Haftantritt noch nicht einmal den Hauptschulabschluss vorweisen konnten, zurückging. Wenn der Anteil der Jugendlichen ohne Hauptschulabschluss außerhalb der Gefängnismauern stetig sinkt und das Übergangssystem vielen Schülern, die in den allgemeinbildenden Schulen gescheitert sind, noch zur Berufsreife in Form des Hauptschulabschlusses verhilft, dann steigt auch der Anteil der Jugendstrafgefangenen mit Hauptschulabschluss in den Vollzugsanstalten. Hinzu kommt die Entwertung des Hauptschulabschlusses. War früher ein ordentlicher Abschluss der Hauptschule die Eintrittskarte in das Berufsleben und mit sozioökonomischer Integration sowie weitgehender gesellschaftlicher Akzeptanz verbunden, bleiben Hauptschulabsolventen heute im beruflichen und sozialen Leben eher ausgeschlossen. Dabei wirkt diese Exklusion und Stigmatisierung als Bildungsverlierer und Leistungsversager beim Zusammentreffen mit weiteren Persönlichkeitseigenschaften und sozioökonomischen Bedingungen eher kriminalitätsfördernd als kriminalitätshemmend. Dass ein Zusammenhang zwischen fehlender Berufsausbildung, drohender Arbeitslosigkeit und kriminellem Verhalten besteht, ergibt sich beispielsweise aus den oben erwähnten Ausführungen von Thomas Feltes, der Rückfalluntersuchung von Karl-Heinz Baumann ${ }^{49}$ und dem Düsseldorfer Gutachten zu den Leitlinien wirkungsorientierter Kriminalprävention, das hierzu ausführt: „Für den Zusammenhang zwischen Arbeitslosigkeit und Kriminalität sind monokausale Zusammenhänge zwar nicht bewiesen, aber unter bestimmten Konstellationen tritt ein kriminalitätsfördernder Effekt ein. “ ${ }^{50} \mathrm{Da}$ Hauptschulabsolventen kaum noch Aussichten auf einen zukunftssichernden Ausbildungsplatz haben, hat das Erreichen der Berufsreife in Form des Hauptschulabschlusses allein wenig bis kaum kriminalitätsmindernde Wirkung, so dass auch hierdurch der Anstieg der Jugendlichen mit Hauptschulabschluss in den Vollzugsanstalten zu erklären ist.

Schlussfolgerungen: Aber nicht nur die Hauptschüler haben weniger bis gar keine Chancen mehr, sich in das Berufsleben zu integrieren, auch die Institution Hauptschule

46 Aktionsrat Bildung 2007, 55.

47 Preisendörfer 2008, 51.

48 Preisendörfer, a.a.O., 41.

49 Baumann, ZfStrVo 1/1984, 36; vgl. auch Feltes, WPB 3/1979, 98.

50 Landeshauptstadt Düsseldorf 2002b, 51; vgl. auch Landeshauptstadt Düsseldorf 2002a, $228 \mathrm{ff}$. 
ist am Ende. Schon längst kann die Hauptschule gemessen an den Schülerzahlen im Sekundarbereich nicht mehr als Hauptschule bezeichnet werden; die Mehrheit der Jugendlichen besucht heute das Gymnasium. „Die Hauptschule wird nicht mehr gewollt und nicht mehr gewählt “"51, und zwar von den Eltern, die versuchen, ihr Kind nicht der Hauptschule „auszuliefern“, der mittlerweile der Ruf einer Restschule anhängt. In 11 der 16 Bundesländer existiert die Hauptschule als eigenständige Schulform nicht mehr (oder wurde wie in den neuen Bundesländern erst gar nicht eingeführt). Die Bildungsgänge Haupt- und Realschule werden dort in kooperativen und integrativen Formen wie Mittel- bzw. Oberschulen oder der Realschule plus zusammengeführt. ${ }^{52}$ Angestoßen wurden diese Bildungsreformen durch die für Deutschland niederschmetternden Ergebnisse der ersten PISA-Studie aus dem Jahr 2000.

Wenn nun, wie oben dargestellt, auf der einen Seite der Hauptschulabschluss erheblich an Wert eingebüßt hat und kein Garant mehr für einen gesicherten sozioökonomischen Status in der Zukunft ist, und auf der anderen Seite der Anteil der Jugendstrafgefangenen mit Hauptschulabschluss in den Vollzugsanstalten steigt, dann ist zu fragen, ob und wenn ja wie die Schule im Jugendstrafvollzug auf diese Veränderungen reagiert hat, um ihrem Resozialisierungsauftrag im Rahmen der tertiären Spezialprävention gerecht zu werden, oder ob die Schule im Jugendstrafvollzug der Entwicklung im öffentlichen Schulwesen hinterherhinkt. Dieser Frage soll in Form einer kurzen qualitativen Erhebung zu den angebotenen abschlussbezogenen schulischen Bildungsmaßnahmen in den deutschen Jugendstrafanstalten abschließend nachgegangen werden.

\section{Erhebung zum Angebot schulischer Bildungsmaßnabmen im Jugendstrafvollzug der $B R D$}

Im Oktober 2011 erfolgte eine telefonische Befragung von Kolleginnen/en in den 27 (selbstständigen) bundesdeutschen Jugendanstalten mit dem Ziel, ein Lagebild des schulischen Bildungsangebots im Jugendvollzug zu zeichnen. Ferner wurden die aus den Internetauftritten der einzelnen Vollzugsanstalten verfügbaren Informationen berücksichtigt.

- In sechs Jugendstrafanstalten (JSA Pforzheim, JVA Hahnöfersand, JVA Heinsberg, JSA Wittlich, Jugendanstalt Schleswig/Neumünster und JSA Ichtershausen) führen die schulischen Bildungsmaßnahmen zum (einfachen) Hauptschulabschluss, weiterführende schulische Bildungsmaßnahmen werden dort nicht angeboten. In den 21 anderen Jugendanstalten existieren über den einfachen Hauptschulabschluss hinausgehende schulische Bildungsangebote, wie der qualifizierende bzw. der erweiterte Hauptschulabschluss, die erweiterte Berufsbildungsreife, das erste Berufsfachschuljahr und der mittlere Bildungsabschluss. Den Erwerb des mittleren Bildungsabschlusses in Form des Realschulabschlusses, der Fachoberschulreife bzw. des mittleren

51 Rösner 2007, 200.

52 Vgl. auch CDU Deutschland, Beschluss Bildungsrepublik Deutschland. 24. Parteitag der CDU Deutschland, 13. - 15.11.2011, 17 (zuletzt besucht am 15.06.2013).

NK 25. Jg. 3/2013 
Schulabschlusses bieten die Länder Berlin, Brandenburg, Baden-Württemberg, Hessen, Niedersachsen und Nordrhein-Westfalen in ihrem Jugendvollzug an. In Bayern besteht hierzu für die Jugendstrafgefangenen die Möglichkeit über die Verlegung in den Erwachsenenvollzug. In der JVA Wriezen/Brandenburg gibt es für besonders befähigte Jugendstrafgefangene das Angebot, sich bis zur Befähigung zum Besuch der gymnasialen Oberstufe zu qualifizieren. In der JVA Iserlohn/NRW kann über die Teilnahme an der externen Prüfung der Fachoberschule des Märkischen Kreises sogar die Fachhochschulreife erworben werden; in Bayern kann ein Online-Studium an der Fernuniversität Hagen absolviert werden. Abschlüsse der Förderschule können in der Jugendanstalt Hameln und der JVA Neumünster erworben werden. Die genannten schulischen Bildungsmaßnahmen werden in Kooperation mit allgemeinbildenden bzw. beruflichen Schulen vor Ort oder über anstaltseigene Kurse mit externen Nichtschülerprüfungen durchgeführt.

- Die Behauptung, dass der Mädchenvollzug innerhalb des Strafvollzuges eine Marginalstellung innehabe ${ }^{53}$, bestätigt sich: Es gibt keine eigene Anstalt für weibliche Jugendstrafgefangene, einige Länder haben die jungen Frauen in speziellen (Jugend-) Abteilungen der Frauenvollzugsanstalten untergebracht. Brandenburg, Bremen, Hamburg, Saarland, Sachsen-Anhalt, Schleswig-Holstein und Thüringen verlegen die straffälligen jungen Frauen über Kooperationsabkommen in den Frauenvollzug der Nachbarländer. Die weiblichen Jugendstrafgefangenen können in den Frauenanstalten an den dort angebotenen schulischen Bildungsmaßnahmen teilnehmen und den Hauptschulabschluss, zum Teil auch einen mittleren Bildungsabschluss, erwerben. Mecklenburg-Vorpommern, Nordrhein-Westfalen und Sachsen bieten dabei koedukativen Unterricht mit männlichen Strafgefangenen an, was nach Aussage der befragten Kolleginnen/en bisher zu keinen nennenswerten Problemen geführt hat.

- Es ist anzumerken, dass die zu mittleren Bildungsabschlüssen bzw. zu qualifizierenden und erweiterten Hauptschulabschlüssen führenden schulischen Bildungsmaßnahmen in den jeweiligen Anstalten in Konkurrenz zu den dortigen Berufsausbildungen treten. Besonders handwerklich begabte und interessierte Inhaftierte werden sich eher zur Teilnahme an einer beruflichen Ausbildungsmaßnahme entscheiden, wenn sie hierfür geeignet sind, sie also z. B. die Berufsreife in Form des Hauptschulabschlusses besitzen und die erforderliche Haftzeit zumindest bis zum Abschluss von Teilmodulen mitbringen. Der Vorteil der Berufsausbildung liegt in der anschließenden direkten Verwertbarkeit bei der Arbeitsplatzsuche nach Entlassung und der Tatsache, dass ein erfolgreicher Berufsabschluss in vielen Ländern als dem mittleren Bil-

53 Vgl. hierzu: Walkenhorst, APuZ 7/2010, 27 sowie ausführlicher Jansen 2007, 238 ff. 
dungsabschluss gleichwertig anerkannt werden $\mathrm{kann}^{54}$. Die für die schulischen Bildungsmaßnahmen vorgehaltenen Plätze und deren Relation zur Belegungskapazität können von daher nicht als Gradmesser für die Qualität des Bildungsangebotes in den einzelnen Vollzugsanstalten herangezogen werden, hierzu müsste deren Verankerung in den umfassenden Bildungskonzeptionen der jeweiligen Jugendstrafanstalt berücksichtigt werden und es bedürfte einer Totalerhebung über alle Bildungskonzepte der Vollzugsanstalten bzw. der Bundesländer. So gibt es Jugendanstalten mit nur wenigen Schulplätzen, aber einem großen Ausbildungsangebot; während andere Anstalten viele schulische Qualifizierungsmaßnahmen vorhalten und die Berufsausbildung in diesen Ländern zentralisiert durchgeführt wird.

- Fazit: Über drei Viertel der bundesdeutschen Jugendstrafanstalten bieten über den einfachen Hauptschulabschluss hinaus weiterführende schulische Maßnahmen an, wobei sich in dem einzelnen Maßnahmetyp das schulpolitische System des jeweiligen Bundeslandes widerspiegelt. Es scheint so, dass die Schule im Jugendvollzug sowohl auf den verbesserten Vorbildungsstand der Jugendstrafgefangenen als auch auf die zunehmende Entwertung des Hauptschulabschlusses reagiert hat. Der Vorwurf, dass die Schule im Jugendvollzug der Entwicklung im übrigen Schulwesen hinterherhinke, kann nach dem hier erhobenen Lagebild der schulischen Maßnahmen im bundesdeutschen Jugendvollzug so nicht aufrechterhalten werden, zaghafte Neuausrichtungen sind erkennbar.

\section{Schlussbetrachtungen}

Sowohl anhand einer empirischen Trendstudie als auch durch Auswertung wissenschaftlicher, literarischer Quellen konnte nachgewiesen werden, dass sich in den letzten Jahrzehnten die Vorbildung der jungen Inhaftierten zu Haftbeginn stetig verbessert hat. Mittlerweile verfügt bei Haftantritt ungefähr die Hälfte der Jugendstrafgefangenen (formell) über die Berufsreife in Form des Hauptschulabschlusses. Gleichzeitig hat der Hauptschulabschluss bildungs- und sozialpolitisch eine totale Entwertung erfahren, er ist längst kein Garant mehr für den Erhalt eines Ausbildungs- oder Arbeitsplatzes. Die Hauptschule ist Restschule, ihre Schüler sind im Wettrennen um eine zukunftssichernde Beschäftigung von Realschülern und Gymnasiasten hoffnungslos abgehängt worden und werden zu einem bildungsfernen KoZ = Kind ohne Zukunft. Das öffentliche Schulwesen hat reagiert: In 11 der 16 Bundesländer gibt es die Hauptschule als eigenständige Schulform nicht bzw. nicht mehr, stattdessen setzt die Schulpolitik auf kooperative und integrative Formen in einem 2-gliedrigen durchlässigen Schulsystem. Diese Entwicklung im

54 Gleichwertigkeit zum qualifizierten Sekundarabschluss I liegt z. B. vor bei einem Notendurchschnitt im Berufsschulabschlusszeugnis von mindestens 3,0 und einer abgeschlossenen Berufsausbildung in einem anerkannten Ausbildungsberuf sowie dem Nachweis ausreichender Fremdsprachenkenntnisse aus mindestens fünf Jahren Fremdsprachenunterricht; vgl. hierzu z. B. $\$ 9$ Berufsschulverordnung Rheinland-Pfalz (BerSchulO RP 2005) vom 07.10.2005 sowie in ähnlicher Form $\ 2$ Verordnung über die Gleichwertigkeit von Abschlüssen im Bereich der beruflichen Bildung Niedersachsen (BB-GVO) vom 19.07.2005.

NK 25. Jg. 3/2013 
öffentlichen Schulwesen darf die Schule im Jugendstrafvollzug nicht „verschlafen“, sie muss den geänderten Vorbildungsstand der ihr zugewiesenen Jugendlichen und Heranwachsenden berücksichtigen und ihr pädagogisches Setting neu ausrichten. Wie dargelegt sind zaghafte Neuausrichtungen der Schule im Jugendvollzug erkennbar: Drei Viertel der bundesdeutschen Jugendstrafanstalten bieten über den einfachen Hauptschulabschluss hinausgehende Bildungsmaßnahmen an, in sieben Bundesländern ist der Erwerb eines mittleren Bildungsabschlusses im Jugendvollzug über die Teilnahme an rein schulischen Bildungsmaßnahmen möglich. Hier besteht für die Zukunft zumindest Prüfungsbedarf: Der Jugendvollzug in den einzelnen Ländern muss sich fragen, ob in seinen Bildungskonzepten die wachsende Klientel der inhaftierten Jugendlichen und Heranwachsenden mit Hauptschulabschluss angemessen berücksichtigt wird, um auch diesen Jugendstrafgefangenen entweder über schulische oder über berufliche Bildungsmaßnahmen weiterführende Abschlüsse zu ermöglichen, die ihnen nach der Entlassung eine reelle Chance auf den Erhalt einer zukunftssichernden Beschäftigung geben. Herbert Hilkenbach, Ehrenvorsitzender der Bundesarbeitsgemeinschaft der Lehrerinnen und Lehrer im Justizvollzug, wendet sich 1987 noch gegen höhere Schulabschlüsse im Strafvollzug, wenn er fragt, ob „es richtig ist, die Gefangenen, die jede Schulförderung annehmen und anstreben, immer weiter zu qualifizieren, d. h. zu höheren Abschlüssen zu bringen " 55 und spricht hinsichtlich der Erfüllung der Bildungswünsche von Gefangenen von einem "Gefälligkeitsvollzug“. ${ }^{56}$ Dieser Vorwurf ist heute nach 25 Jahren nicht mehr angebracht. Natürlich ist die Förderung der Elementar- und Allgemeinbildung über die nicht abschlussbezogenen schulischen Bildungsmaßnahmen im Vollzug auch im Hinblick auf einen später angestrebten Schulabschluss bzw. eine Berufsausbildung wichtig und unabdingbar. Aber heute wird es Gefangenen oft nur mit weiterführenden Schulabschlüssen möglich sein - um wiederum Hilkenbach zu zitieren - „selbständig ihr Leben zu führen bzw. in der Arbeitswelt Fuß zu fassen. “" ${ }^{57}$ Mit einer verbesserten Allgemeinbildung oder einem (mäßigen) Hauptschulabschluss wird ihnen das allein nicht gelingen. Die genannten Evaluationsstudien schulischer Bildungsmaßnahmen im Vollzug geben Hilkenbach Recht, wenn er feststellt, dass „Maßnahmen, die zu Schulabschlüssen führen, danach allein offenbar wenig hilfreich sind, das Ziel des Vollzuges zu erreichen " ${ }^{58}$, und folgerichtig fordert er „im Einzelfall sehr genau zu prüfen, ob nicht eine [sic] Qualifizierung auf beruflicher Ebene der weiteren schulischen Bildung der Vorrang zu geben ist. " 59 Dies gilt auch heute noch, und die Bildungskonzeptionen der einzelnen Länder bzw. Jugendvollzugsanstalten sind bezüglich des Angebotes und der Verzahnung schulischer und beruflicher Bildungsmaßnahmen stetig zu evaluieren. Dabei ist zu bedenken, dass heute oft erst ein guter weiterführender Schulabschluss die Basis für eine spätere berufliche Bildungsmaßnahme im Vollzug oder nach Entlassung ist.

55 Hilkenbach, ZfStrVo 1/1987, 51.

56 Hilkenbach, a.a.O., 51.

57 Hilkenbach, a.a.O., 51.

58 Hilkenbach, a.a.O., 51.

59 Hilkenbach, a.a.O., 51.

TITEL 
Die Schule im Jugendstrafvollzug vermag hier ihren Beitrag zum Aufbau eines entsprechenden sozialen Kapitals zu leisten, wenn sie bereits im Vollzug oder im Anschluss nach der Entlassung den Weg in eine zukunftssichernde Berufsausbildung und den Ausstieg aus der kriminellen Karriere ebnet, wie die amerikanischen Entwicklungskriminologen Sampson und Laub darlegen: „[... ] desistance from criminal behavior in adulthood can be explained by strong social bonds in adulthood, despite a background of delinquent behavior." 60 In der kritischen Phase nach der Entlassung kann der Jugendstrafgefangene die Chance ergreifen, unterstützt durch das Coaching des vollzuglichen Übergangsmanagements, im Berufsleben Fuß zu fassen und entsprechend starke soziale Bindungen aufzubauen. Hier ist der Beitrag der schulischen Bildungsmaßnahmen im Rahmen der tertiären Spezialprävention zu sehen. Sollte sich der entlassene Jugendliche jedoch anderen Gruppen mit schwacher sozialer Kontrolle zuwenden, ist bei einem Rückfall oft der Weg in den Erwachsenenvollzug die Folge. Das eingangs erwähnte AchternbuschZitat ist abzuändern:

$$
\text { „Ergreife deine Chance im Jugendvollzug, denn es ist deine letzte. “ }
$$

\section{Literatur}

Achternbusch, H. (1986), Die Atlantikschwimmer. Schriften 1973-79, 1. Aufl.

Aktionsrat Bildung (2007), Bildungsgerechtigkeit. Jahresgutachten 2007, 1. Aufl.

Autorengruppe Bildungsberichterstattung (Hrsg.) (2010), Bildung in Deutschland 2010. Ein indikatorengestützter Bericht mit einer Analyse zu Perspektiven des Bildungswesens im demografischen Wandel

Baumann, K.-H. (1984), Der Einfluß von Bildungsmaßnahmen im Strafvollzug auf das Rückfallverhalten, in:Zeitschrift für Strafvollzug und Straffälligenhilfe (ZfStrVo) 1/1984, $31-36$

Baumert, J. (2001), PISA 2000. Basiskompetenzen von Schülerinnen und Schülern im internationalen Vergleich

Berckbaner, F. / Hasenpusch, B. (1982), Legalbewährung nach Strafvollzug. Zur Rückfälligkeit der 1974 aus dem niedersächsischen Strafvollzug Entlassenen, in: Schwind / Steinhilper (Hrsg.), Modelle zur Kriminalitätsvorbeugung und Resozialisierung. Beispiele praktischer Kriminalpolitik in Niedersachsen, S. 281-359

CDU Deutschland: Beschluss Bildungsrepublik Deutschland. 24. Parteitag der CDU Deutschland. 13.- 15.11.2011 Leipziger Messe, http://www.leipzig2011.cdu.de/images/ stories/docs/111115-beschluss-bildungsrepublik-deutschland.pdf, zuletzt besucht am 15.06.2013

NK 25. Jg. 3/2013 
Diekmann, A. (2010), Empirische Sozialforschung. Grundlagen, Methoden, Anwendungen, 4. Aufl.

Dolde, G. / Grübl, G. (1996), Jugendstrafvollzug in Baden-Württemberg. Untersuchungen zur Biographie, zum Vollzugsverlauf und zur Rückfälligkeit von ehemaligen Jugendstrafgefangenen, in: Kerner / Dolde / Mey (Hrsg.), Jugendstrafvollzug und Bewährung. Analysen zum Vollzugsverlauf und zur Rückfallentwicklung, S. 219-356

Dünkel, F. / Pörksen, A. (2007), Stand der Gesetzgebung zum Jugendstrafvollzug und erste Einschätzungen, in: Neue Kriminalpolitik (NK) 2/2007, S. 55-68

Eberle, H.-J. (1980), Lernen im Justizvollzug. Voraussetzungen und Ansätze einer Justizvollzugspädagogik und ihrer Didaktik

Feltes, T. (1979), Jugendkriminalität und Schule. Zum Zusammenhang von Schulbildung, Sozialstatus und abweichendem Verhalten: Konsequenzen für die Schule, in: Westermanns Pädagogische Beiträge (WPB) 3/1979, S. 96-101

Giebel, S. M., "Rückfälligkeit" bei jugendlichen Straftätern in den Jahrgängen Ende 1996 - Anfang 2000, http://sma.uni.lu/stat2/files/Giebel.pdf, zuletzt besucht am 15.06.2013

Großkelwing, G. (1976), Schulische und berufliche Bildung, in: Schwind / Blau (Hrsg.), Strafvollzug in der Praxis. Eine Einführung in die Probleme und Realitäten des Strafvollzuges und der Entlassenenhilfe, S. 297-304

Hammermann, H. A. (1977), Die Schul- und Berufsausbildung von Gefangenen, in: Zeitschrift für Strafvollzug und Straffälligenhilfe (ZfStrVo) 1977, S. 131-137

Hilkenbach, H. (1979), Schulische und berufliche Bildung im Jugendstrafvollzug, in: Zeitschrift für Strafvollzug und Straffälligenhilfe (ZfStrVo) 2/1979, S. 83-89

Hilkenbach, H. (1987), Schule und berufliche Bildung im Strafvollzug - seit Inkrafttreten des Strafvollzugsgesetzes -, in: Zeitschrift für Strafvollzug und Straffälligenhilfe (ZfStrVo) 1/1987, S. 49-52

Hoffmann, M. (1989), Unterricht im Strafvollzug, in: Goetze / Neukäter (Hrsg.), Pädagogik bei Verhaltensstörungen. Handbuch der Sonderpädagogik, Bd. 6, S. 464-472

Jansen, I. (2007), Gender Mainstreaming im Jugendstrafvollzug, in: Goerdeler / Walkenhorst (Hrsg.), Jugendstrafvollzug in Deutschland. Neue Gesetze, neue Strukturen, neue Praxis?, S. 238-253

Jeble, J.-M. / Albrecht, H.-J. / Hohmann-Fricke, S. / Tetal, C. ( (2010), Legalbewährung nach strafrechtlichen Sanktionen. Eine bundesweite Rückfalluntersuchung 2004 bis 2007,1 . Aufl.

Klemm, K., Jugendliche ohne Hauptschulabschluss. Analysen - Regionale Trends - Reformansätze, http://www.bertelsmann-stiftung.de/bst/de/media/xcms_bst_dms_323 43_32344_2.pdf, zuletzt besucht am 15.06.2013 
Konsortium Bildungsberichterstattung (Hrsg.) (2006), Bildung in Deutschland. Ein indikatorengestützter Bericht mit einer Analyse zu Bildung und Migration

Krohne, K. (1889), Lehrbuch der Gefängniskunde unter Berücksichtigung der Kriminalstatistik und Kriminalpolitik

Kruse, H.-J. (1994), Schulqualifikation für junge Knackis - Chance oder Alibi? Ein Bericht, in: Unsere Jugend - Zeitschrift für Studium und Praxis der Sozialpädagogik 5/1994, S. $189-197$

Kruse, H.-J. (1999), Berufsausbildung und Schule im Bremer Jugendvollzug (1945 - 1998)

Landeshauptstadt Düsseldorf - Arbeitskreis Vorbengung und Sicherbeit (Hrsg.) (2002a), Düsseldorfer Gutachten: Empirisch gesicherte Erkenntnisse über kriminalpräventive Wirkungen. Eine Sekundäranalyse der kriminalpräventiven Wirkungsforschung; Gutachten für die Landeshauptstadt Düsseldorf

Landeshauptstadt Düsseldorf - Arbeitskreis Vorbengung und Sicherheit (Hrsg.) (2002b), Düsseldorfer Gutachten: Leitlinien wirkungsorientierter Kriminalprävention

Lang, H. (2001), Vorbereitung auf den Qualifizierenden Hauptschulabschluss während der Untersuchungshaft, in: Zeitschrift für Strafvollzug und Straffälligenhilfe (ZfStrVo) 3/2001, S. $152-155$

Liszt, F. von (1905), Strafrechliche Aufsätze und Vorträge, Zweiter Band. 1892 - 1904

Matt, E. / Maul, K. (2005), Das Problem der Heterogenität der Klientel für Bildungsmaßnahmen einer Justizvollzugsanstalt, in: Zeitschrift für Strafvollzug und Straffälligenhilfe (ZfStrVo) 4/2005, S. 198-202

Myschker, N. (2009), Verhaltensstörungen bei Kindern und Jugendlichen. Erscheinungsformen - Ursachen - hilfreiche Maßnahmen, 6., überarb. und aktualisierte Aufl.

Myschker, N. / Hoffmann, K. (1984), Unterricht mit jugendlichen Inhaftierten

Oblendorf, V., Schulabschluss im Gefängnis. Bericht über die JSA Regis-Breitingen, http://mephisto976.uni-leipzig.de/startseite/gesellschaft/beitrag/artikel/schulabschluss-im-gefaengnis.html, zuletzt besucht am 15.06.2013

Ostendorf, H. (2007), Das Ziel des Jugendstrafvollzugs nach zukünftigen Recht, in: Goerdeler / Walkenhorst (Hrsg.), Jugendstrafvollzug in Deutschland. Neue Gesetze, neue Strukturen, neue Praxis?, S. 100-113

Preisendörfer, B. (2008), Das Bildungsprivileg. Warum Chancengleichheit unerwünscht ist

Reinheckel, S. (2013), Geringqualifikation bei männlichen Strafgefangenen im geschlossenen Jugendstrafvollzug der Bundesrepublik Deutschland - eine empirische Untersuchung, http://nbn-resolving.de/urn:nbn:de:0168-ssoar-349418, zuletzt besucht am 20.08.2013

NK 25. Jg. 3/2013 
Rösner, E. (2007), Hauptschule am Ende. Ein Nachruf

Sampson, R. J. / Laub, J. H. (1993), Crime in the making. Pathways and turning points through life

Schmid, A. (1964), Bildungsmittel und allgemeiner Intelligenzgrad bei jungen Strafgefangenen, in: Zeitschrift für Strafvollzug: für Praxis und Wissenschaft 6/1964, S. 336-338

Sessar-Karpp, E. (1982), Lernvoraussetzungen jugendlicher Inhaftierter. Eine Studie zum Überdenken der gegenwärtigen Unterrichtspraxis im Jugendjustizvollzug

Sonnen, R.-B. (2007), Fördern, Fordern, Fallen lassen, in: Neue Kriminalpolitik (NK) $2 / 2007$, S. $50-54$

Statistisches Bundesamt, Fachserie 11 Reihe 1. Bildung und Kultur. Allgemeinbildende Schulen Schuljahr 2011/2012,

https://www.destatis.de/DE/Publikationen/Thematisch/BildungForschungKultur/ Schulen/AllgemeinbildendeSchulen2110100127004.pdf?_blob=publicationFile, zuletzt besucht am 15.06.2013

Statistisches Landesamt Rheinland-Pfalz: Allgemeinbildende Schulen im Schuljahr 2010/11, Teil I, http://www.statistik.rlp.de/fileadmin/dokumente/berichte/ B1013_201000_1j_K.pdf, zuletzt besucht am 15.06.2013

Stelly, W. / Thomas, J. (2011), Die sozialen Lebenslagen von Jugendstrafgefangenen, in: Stelly / Thomas (Hrsg.), Erziehung und Strafe. Symposium zum 35-jährigen Bestehen der JVA Adelsheim, 1. Aufl., S. 127-144

Stelly, W. / Walter, J. (2011), Russlanddeutsche im Jugendstrafvollzug - was ist aus ihnen geworden?, in: Neue Kriminalpolitik (NK) 2/2011, S. 50-54

Walkenhorst, P. (2002), "Gute Schulen" im Jugendstrafvollzug - Jugendstrafvollzug als "gute Schule". Überlegungen zu Voraussetzungen und Möglichkeiten, in: Bereswill / Höynck (Hrsg.), Jugendstrafvollzug in Deutschland: Grundlagen, Konzepte, Handlungsfelder. Beiträge aus Forschung und Praxis, S. 319-355

Walkenhorst, P. (2010), Jugendstrafvollzug, in: Aus Politik und Zeitgeschichte (APuZ) 7/2010, S. $22-28$

Walter, J. (2011), Jugendstrafvollzug heute und morgen, in: Stelly / Thomas (Hrsg.), Erziehung und Strafe. Symposium zum 35-jährigen Bestehen der JVA Adelsheim, 1. Aufl., S. 95-117

Wichern, J. H. (1973), Erlaß wegen des Schulunterrichts in den Strafanstalten (1860), in: Meinhold (Hrsg.), Johann Hinrich Wichern. Sämtliche Werke. Band VI: Die Schriften zur Gefängnisreform, S. 179-188 
Wirth, W. (2009), 3-Säulenstrategie zur beruflichen Reintegration von Gefangenen. Erfahrungen mit einem systematischen Übergangsmanagement, in: Forum Strafvollzug (FS) 2/2009, S. 75-84

Wirth, W. (2007), Qualifizierung - Vermittlung - Nachsorge. Eine 3-Säulen-Strategie zur beruflichen Wiedereingliederung von (ehemaligen) Strafgefangenen, in: Zeitschrift für soziale Strafrechtspflege 44/2007, S. 13-27

Zelinka, A. G. (2010), Prävalenz der Aufmerksamkeitsdefizit-/Hyperaktivitätsstörung bei männlichen jugendlichen Delinquenten im Strafvollzug in verschiedenen Deliktgruppen, zugleich medizinische Dissertation

ZUM-Wiki, Schule in der JVA, http://wiki.zum.de/Schule_in_der_JVA, zuletzt besucht am 15.06.2013

Jens Gudel M.A.

Kriminologe und Oberlebrer

Jugendstrafanstalt Schifferstadt

Rudolf-Diesel-Str. 15

67105 Schifferstadt

Tel.: 06235 / 499-1702

e-mail: jens.gudel@vollzug.mjv.rlp.de 